\title{
Peripapillary Vessel Density Measurement of Quadrant and Clock-Hour Sectors in Primary Angle Closure Glaucoma Using Optical Coherence Tomography Angiography
}

\section{YongDong Lin}

Joint Shantou International Eye Center of Shantou University and The Chinese University of Hong Kong

\section{ShiRong Chen}

Joint Shantou International Eye Center of Shantou University and The Chinese University of Hong Kong MingZhi Zhang ( $\sim$ Zmz0754@126.com)

Joint Shantou International Eye Center of Shantou University and The Chinese University of Hong Kong

\section{Research Article}

Keywords: optical coherence tomography angiography, peripapillary vessel density, primary angle closure glaucoma

Posted Date: January 4th, 2021

DOl: https://doi.org/10.21203/rs.3.rs-128309/v1

License: (c) (1) This work is licensed under a Creative Commons Attribution 4.0 International License.

Read Full License 


\section{Abstract}

Background: The purpose of this study was to investigate peripapillary vessel density of primary angle closure glaucoma (PACG) eyes in quadrant and clock-hour sectors, and the diagnostic ability of peripapillary vessel density was also studied.

Methods: This was a cross-sectional study on forty-one PACG patients(50 eyes)and twenty-seven healthy subjects(27 eyes). The optic disc was imaged using a 1050-nm-wavelength swept-source optical coherence tomography system (DRI OCT Triton, TOPCON). Peripapillary vessel density was quantified by imageJ software. Characteristics of peripapillary vessel density of quadrant and clock-hour sectors were analyzed, and diagnostic capability was evaluated by areas under the receiver operating characteristics curves (AUCs).

Results: Compared to the control group, four quadrants and each clock-hour sectors of peripapillary vessel density of glaucomatous group decreased to different degrees, and vessel density reduced most at 7 o'clock. Except 4 o'clock, there was not any statistical difference between the diagnostic ability of peripapillary vessel density and peripapillary retinal nerve fiber layer thickness. The inferior quadrant peripapillary vessel density had the best diagnostic value (AUCs0.944), followed by 7 o'clock vessel density (AUCs0.937), average vessel density (AUCs0.926) and 7 o'clock RNFL thickness (AUCs0.922).

Conclusions: In PACG, the diagnostic ability of peripapillary vessel density is equivalent to peripapillary retinal nerve fiber layer thickness. Understanding spatial characteristics of peripapillary vessel density in PACG may be helpful for clinical diagnosis and treatment.

\section{Background}

There is increasing evidence show that vascular factors are closely related to the pathogenesis of glaucoma[1].In the past, there was a lack of effective non-invasive instruments to study the vascular mechanism of glaucoma.[2-5].Recently, research on glaucoma by optical coherence tomography angiography (OCTA)showed that the peripapillary vessel density decreased and the peripapillary vessel density had good diagnostic ability for glaucoma [5, 6].Previous articles used OCTA to study the spatial characteristics of peripapillary vessel density in glaucoma mainly on average and quadrants, and articles focusing on clock-hour peripapillary vessel density were limited[7, 8]. As far as we know, there is currently no article on peripapillary clock-hour vessel density of primary angle closure glaucoma (PACG). Previous studies have proved that the changes of neuroretinal rim in the eyes of glaucoma patients can be diffuse or local. Therefore, understanding the clock-hour spatial characteristics of peripapillary vessel density may help us to further understand the vascular mechanism of glaucoma and improve the diagnostic ability for glaucoma.

We know little about whether optic disc capillary atrophy in glaucoma eye is diffuse or localized. Shin et al. [9] studied peripapillary vessel density in eyes of normal-tension glaucoma(NTG) at clock-hour sectors using OCTA. They found that the superficial and deep vessel density of glaucoma at 7 and 11 o'clock 
positions decreased the most compared with healthy eyes. The diagnostic ability of 7o'clock position was the highest. The mechanism of PACG is different from NTG and primary open angle glaucoma(POAG) [10].We do not know whether the peripapillary vessel density is the same for PACG.

Therefore, the purpose of this study was to use OCTA to study the quadrant and clock-hour spatial characteristics of peripapillary vessel density in PACG, and also to assess the diagnostic ability of vessel density.

\section{Materials And Methods}

\section{Study subjects}

We told each subject the content of the study. Their willingness to participate was recorded and they voluntarily signed the consent form approved by the institutional ethics committee. PACG patient and healthy person were selected from October 2018 to October 2019. All subjects underwent a detailed medical history, best corrected visual acuity (BCVA), intraocular pressure(IOP) measurement, axial length measurement by OA-2000 (Tomey $\mathrm{GmbH}$, Nagoya, Japan), fundus examination, and swept-source optical coherence tomography (OCT) (DRI OCT Triton, TOPCON) examination. Systolic blood pressure (SBP) and diastolic blood pressure(DBP) of subjects were measured before OCTA was measured. The ocular perfusion pressure (OPP) was calculated as the following formula:

$$
O P P=2 / 3(\mathrm{DBP}+0.42(\mathrm{SBP}-\mathrm{DBP}))-\operatorname{IOP}[11]
$$

Glaucoma patients also underwent visual field (VF)examination by the static automated white-on-white threshold 24-2 SITA standard strategy (Humphrey Field Analyzer II; Carl Zeiss Meditec) . Our study only included reliable visual field examination results (i.e., false-negative errors $<15 \%$, false-positive errors $<15 \%$, and fixation loss $<20 \%$ )

The diagnostic criteria for PACG were as follows[12]: 1.the presence of an occluded angle defined as an angle in which $>270^{\circ}$ of the posterior trabecular meshwork cannot be seen, as verified with gonioscopy. 2 . IOP of more than $21 \mathrm{mmHg}$. 3. optic disc has RNFL damage due to glaucoma. Inclusion criteria for healthy eyes:1. age: $>18$ years old. 2 . normal anterior segment and fundus in clinical examination by experts.3. intraocular pressure $\leq 21 \mathrm{mmmhg}$. 4. no family history of glaucoma. Exclusion criteria for all participants were: 1 . diopter $\geq 6.0 \mathrm{D}$ (sphere) and or $3.0 \mathrm{D}$ (cylinder). 2. previous eye surgery and other eye diseases. 3. poor OCT or OCTA image quality score (less than 40 points).

\section{OCTA imaging acquisition and processing}

All subjects were examined by $4.5 \times 4.5 \mathrm{~mm}$ OCTA optic disc scans (DRI OCT Triton; Topcon Corporation, Tokyo, Japan).Images were analyzed by an A-scan rate of 100,000 scans per second, wavelengthscanning light centered on 1,050nm, and in-depth digital resolution of $2.6 \mathrm{~mm}[13]$. We used OCT angiography ratio analyses(OCTARA) of the device to generate the maps. The system automatically 
divided the optic disc into four layers, and the selected layer was Nerve Head layer. Nerve Head layer is defined as 130 um below the internal limiting membrane. Peripapillary vessel density measurement was calculated in the Nerve Head layer.

We reviewed and filtered images quality after each scan. Images with significant motion artifacts, poor signal strength (signal strength index $<40$ ), or poor image clarity were discarded. We performed the Phansalkar as adaptive local thresholding methods[14]. This method was used for binarization algorisms in OCTA images using Image $\mathrm{J}$ software (National Institutes of Health, Bethesda, MD) to obtain vascular signals as a white region and digitize this area. The vessel density value was defined as a proportion of vessel signal in area of interest[15]. The peripapillary region was $750 \mathrm{~mm}$-wide annular region of interest centered on the optic disc, with an inner diameter of $1.95 \mathrm{~cm}$ and an outer diameter of $3.45 \mathrm{~cm}[16]$. The peripapillary region was divided into 4 equal quadrants, namely superior, inferior, temporal and nasal quadrants. The peripapillary region also was divided into the 12 clock-hour sectors. OCTA image processing steps were shown in Fig 1.

\section{Statistical Analyses}

Data analysis was carried out using commercially available software (SPSS ver.13.0; SPSS Inc, Chicago, Illinois, USA) and MedCalc(ver.15.2.2, Mariakerke, Belgium). We used mean \pm standard and ratios to describe all data and the Shapiro-Wilk test to evaluate the normal distribution of continuous variables. Independent $t$ test and Mann-Whitney test were used to observe the differences between the PACG eyes and healthy eyes. We calculated and compared the areas under the receiver operating characteristics curves (AUCs) of average quadrant and clock-hour vessel density and peripapillary retinal nerve fiber layer (RNFL) to evaluate the diagnostic ability for glaucoma. Pearson correlation analysis and Spearman correlation test were used to evaluate the correlation between vessel density parameters and related factors. P-value of $<0.05$ was taken to be of statistical significance.

\section{Results}

Forty-eight PACG patients $₫ 60$ eyes $₫$ and 33 healthy people $₫ 33$ eyes $₫$ were enrolled in this study. Ten eyes of PACG patients and 6 eyes of healthy subjects were excluded due to poor OCTA image quality. Therefore, 41 PACG patients (50 eyes) and 27 healthy subjects (27 eyes) were enrolled. Demographics and clinical characteristics of study subjects are listed in Table 1. The axial length of PACG eyes was significantly different from that of healthy eyes(pष0.001), and there were no significant differences in age, sex, intraocular pressure at examination and ocular perfusion pressure $(\mathrm{p} \otimes 0.05)$. The $\mathrm{VF}$ mean deviation in PACG eyes was $-18.3 \pm 9.9 \mathrm{db}$.

\section{Table 1 Demographic and clinical characteristics of the study population}




\begin{tabular}{|c|c|c|c|}
\hline & Healthy eyes $(n=27)$ & PACG eyes(n=50) & $P$ value \\
\hline 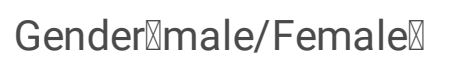 & $7: 20$ & $17: 24$ & $0.190 *$ \\
\hline Age(year) & $61.9 \pm 7.6$ & $62.3 \pm 7.9$ & 0.843 \\
\hline $\mathrm{SBP}(\mathrm{mmHg})$ & $137.6 \pm 12.3$ & $135.6 \pm 20.6$ & $0.877^{\dagger}$ \\
\hline $\mathrm{DBP}(\mathrm{mmHg})$ & $84.8 \pm 7.2$ & $80.7 \pm 10.0$ & $0.074^{\dagger}$ \\
\hline OPP(mmHg) & $57.4 \pm 6.8$ & $54.1 \pm 9.7$ & $0.162^{\dagger}$ \\
\hline BCVA(log MAR) & $0.6 \pm 0.2$ & $0.5 \pm 0.2$ & 0.163 \\
\hline IOP at imaging $(\mathrm{mmHg})$ & $13.9 \pm 2.9$ & $15.1 \pm 3.7$ & $0.087^{\dagger}$ \\
\hline $\mathrm{Al}(\mathrm{mm})$ & $23.6 \pm 0.9$ & $22.4 \pm 1.1$ & $\otimes 0.001^{\dagger}$ \\
\hline $\operatorname{VF~} \mathrm{MD}(\mathrm{dB})$ & - & $-18.3 \pm 9.9$ & - \\
\hline
\end{tabular}

PACG , primary angle closure glaucoma; SBP, systolic blood pressure; DBP, diastolic blood pressure; OPP, ocular perfusion pressure; BCVA, best-corrected visual acuity; IOP, intraocular pressure; AL, axial length; VF $M D$, visual field mean deviation

Unless otherwise illustrated, the comparison was made by using independent sample $t$ test.

*The comparison was performed by using the chi-square test.

${ }^{\dagger}$ The comparison was performed by using the Mann-Whitney test.

Table 2 shows data of peripapillary vessel density and RNFL thickness on the mean average, quadrant, and clock-hour regions. Compared with the control group, the peripapillary vessel density of glaucoma in all measured sectors decreased to different degrees and the difference was significant(P凶0.05), with 7 o'clock decreased most. Except for 3 o'clock (Pख0.05), peripapillary RNFL in all measured sectors showed significant difference between the glaucoma group and control group(P®0.05). Three o'clock sector was a less important sector in glaucomatous diagnosis. Fig 2 shows a decrease in infratemporal and supratemporal peripapillary vessel density sectors of glaucoma and atrophy of RNFL thickness in corresponding regions.

Table 2 Peripapillary retinal nerve fiber layer thickness and peripapillary vessel density in healthy and glaucomatous eyes 


\begin{tabular}{|c|c|c|c|c|c|c|}
\hline \multirow[t]{2}{*}{ Parametres } & \multicolumn{3}{|c|}{ RNFL thickness } & \multicolumn{3}{|c|}{ Peripapillary vessel density } \\
\hline & $\begin{array}{l}\text { Healthy } \\
\text { eyes }(n=27)\end{array}$ & $\begin{array}{l}\text { PACG } \\
\text { eyes }(n=50)\end{array}$ & $\begin{array}{l}P \\
\text { value }\end{array}$ & $\begin{array}{l}\text { Healthy } \\
\text { eyes }(n=27)\end{array}$ & $\begin{array}{l}\text { PACG } \\
\text { eyes }(n=50)\end{array}$ & $\begin{array}{l}P \\
\text { value }\end{array}$ \\
\hline Average & $113.0 \pm 9.6$ & $78.3 \pm 26.1$ & $0.001 *$ & $54.8 \pm 2.5$ & $46.0 \pm 5.6$ & ${ }^{\square} .001 *$ \\
\hline Temporal & $85.4 \pm 10.3$ & $64.0 \pm 18.4$ & $0.001 *$ & $54.8 \pm 5.4$ & $47.1 \pm 6.8$ & $\nabla 0.001$ \\
\hline Superior & $142.7 \pm 19.2$ & $92.9 \pm 38.2$ & $\begin{array}{l}\square \\
0.001 *\end{array}$ & $57.3 \pm 2.7$ & $48.5 \pm 6.6$ & ${ }^{\square} 0.001 *$ \\
\hline Nasal & $76.7 \pm 14.5$ & $65.7 \pm 21.3$ & 0.019 & $49.0 \pm 5.5$ & $42.2 \pm 7.2$ & $\nabla 0.001$ \\
\hline Inferior & $147.6 \pm 12.9$ & $86.8 \pm 40.4$ & ${ }^{0} 0.001 *$ & $58.3 \pm 3.0$ & $46.4 \pm 6.5$ & ${ }^{0} 0.001 *$ \\
\hline 9 & $72.1 \pm 7.9$ & $60.6 \pm 14.4$ & $0.001^{*}$ & $50.3 \pm 7.1$ & $46.4 \pm 6.6$ & 0.018 \\
\hline 10 & $100.1 \pm 14.0$ & $69.0 \pm 26.6$ & $0.001 *$ & $58.4 \pm 4.9$ & $49.5 \pm 7.7$ & 0.001 * \\
\hline 11 & $148.3 \pm 24.8$ & $91.2 \pm 42.1$ & $0.001 *$ & $59.2 \pm 3.6$ & $47.9 \pm 8.9$ & $0.001 *$ \\
\hline 12 & $144.8 \pm 30.8$ & $98.2 \pm 43.2$ & $\varangle 0.001$ & $56.9 \pm 3.6$ & $50.1 \pm 7.3$ & $0.001 *$ \\
\hline 1 & $135.1 \pm 23.5$ & $89.1 \pm 37.5$ & $0.001 *$ & $55.9 \pm 3.9$ & $47.8 \pm 6.9$ & $\begin{array}{l}0.001 * \\
\end{array}$ \\
\hline 2 & $90.9 \pm 22.2$ & $75.4 \pm 28.8$ & 0.018 & $51.0 \pm 5.9$ & $44.4 \pm 8.4$ & $0.001 *$ \\
\hline 3 & $66.7 \pm 14.5$ & $60.4 \pm 19.1$ & 0.137 & $46.7 \pm 7.0$ & $40.6 \pm 8.1$ & 0.001 \\
\hline 4 & $72.5 \pm 12.0$ & $60.2 \pm 22.8$ & $0.003^{*}$ & $49.5 \pm 5.0$ & $41.3 \pm 8.4$ & ${ }^{\square} .001 *$ \\
\hline 5 & $123.1 \pm 16.2$ & $86.2 \pm 38.1$ & 0.001 * & $55.7 \pm 4.7$ & $44.7 \pm 7.9$ & 0.001 * \\
\hline 6 & $157.9 \pm 20.1$ & $102.5 \pm 46.8$ & $\begin{array}{l}\square \\
0.001 *\end{array}$ & $58.7 \pm 4.1$ & $49.0 \pm 6.9$ & ${ }^{\square} 0.001 *$ \\
\hline 7 & $161.3 \pm 25.8$ & $82.5 \pm 43.2$ & 0.001* & $60.4 \pm 4.7$ & $45.3 \pm 8.8$ & $\begin{array}{l}0.001 * \\
\end{array}$ \\
\hline 8 & $82.7 \pm 17.0$ & $62.0 \pm 19.9$ & $0.001 *$ & $55.2 \pm 6.4$ & $45.5 \pm 8.1$ & $\nabla 0.001$ \\
\hline
\end{tabular}

RNFL, retinal nerve fiber layer; PACG , primary angle closure glaucoma

Unless otherwise illustrated, the comparison was made by using independent sample $t$ test. 
*The comparison was performed by using the Mann-Whitney test.

AUCs of peripapillary vessel density was between 0.658 in 9 o'clock sector and 0.944 in the inferior quadrant. AUCs of RNFL thickness was between 0.596 in 3 o'clock sector and 0.922 in 7 o'clock sector. Inferior quadrant vessel density had the highest diagnostic ability (AUCs0.944), followed by 7 clock-hour peripapillary vessel density (AUCs0.937), average vessel density (AUCs0.926) and 7 o'clock RNFL thickness (0.922). Except 4 o'clock, there was no statistical difference between the diagnostic ability of peripapillary vessel density and RNFL. The diagnostic ability of 4 o'clock vessel density was higher than that of RNFL (Table 3).

Table 3 Areas under the receiver operating characteristics curves comparison for glaucomatous discrimination ability between Peripapillary vessel density and RNFL thickness

\begin{tabular}{|c|c|c|c|c|c|}
\hline \multirow{3}{*}{$\begin{array}{l}\text { Parametres } \\
\text { Optic disc }\end{array}$} & \multicolumn{5}{|c|}{ Control and glaucomatous eyes } \\
\hline & \multicolumn{2}{|c|}{ Peripapillary vessel density } & \multicolumn{2}{|c|}{ RNFL thickness } & \multirow[t]{2}{*}{$P$ value } \\
\hline & AUC & $95 \% \mathrm{Cl}$ & AUC & $95 \% \mathrm{Cl}$ & \\
\hline Average & 0.926 & $0.844-0.973$ & 0.885 & $0.792-0.947$ & 0.265 \\
\hline Temporal & 0.814 & $0.709-0.893$ & 0.832 & $0.729-0.907$ & 0.727 \\
\hline Superior & 0.880 & $0.786-0.943$ & 0.859 & $0.760-0.927$ & 0.655 \\
\hline Nasal & 0.769 & $0.659-0.857$ & 0.664 & $0.548-0.768$ & 0.054 \\
\hline Inferior & 0.944 & $0.866-0.983$ & 0.904 & $0.815-0.959$ & 0.290 \\
\hline 9 & 0.658 & $0.541-0.762$ & 0.739 & $0.626-0.832$ & 0.235 \\
\hline 10 & 0.834 & $0.732-0.909$ & 0.843 & $0.743-0.916$ & 0.848 \\
\hline 11 & 0.875 & $0.780-0.939$ & 0.877 & $0.782-0.941$ & 0.968 \\
\hline 12 & 0.796 & $0.688-0.879$ & 0.810 & $0.705-0.890$ & 0.801 \\
\hline 1 & 0.834 & $0.732-0.909$ & 0.856 & $0.757-0.925$ & 0.667 \\
\hline 2 & 0.735 & $0.622-0.829$ & 0.673 & $0.557-0.776$ & 0.202 \\
\hline 3 & 0.714 & $0.600-0.812$ & 0.596 & $0.478-0.706$ & 0.074 \\
\hline 4 & 0.811 & $0.705-0.891$ & 0.675 & $0.558-0.777$ & 0.022 \\
\hline 5 & 0.881 & $0.788-0.944$ & 0.814 & $0.710-0.894$ & 0.161 \\
\hline 6 & 0.881 & $0.787-0.943$ & 0.837 & $0.735-0.911$ & 0.332 \\
\hline 7 & 0.937 & 0.857-0.979 & 0.922 & $0.838-0.971$ & 0.676 \\
\hline 8 & 0.825 & $0.721-0.902$ & 0.787 & $0.679-0.872$ & 0.487 \\
\hline
\end{tabular}


RNFL, retinal nerve fiber layer; AUCs, areas under the receiver operating characteristics curves; $\mathrm{Cl}$, confidence interval;

IOP, average RNFL thickness and VF mean deviation were correlated with average peripapillary vessel density. The P value of IOP was close to 0.05 , indicating that the correlation between average peripapillary vessel density and average RNFL,VF mean deviation were stronger than any other variables (Table 4).

\section{Table 4 The correlation between peripapillary vessel density and glaucomatous related factors}

\begin{tabular}{|lll|}
\hline & \multicolumn{2}{l|}{ Peripapillary vessel density } \\
\hline & $\mathbf{r}$ & $\mathbf{P}$ \\
\hline Age(year) & -0.170 & 0.238 \\
\hline Sex & 0.041 & 0.863 \\
\hline IOP $(\mathrm{mmHg})$ & -0.318 & $0.024^{*}$ \\
\hline Mean OPP $(\mathrm{mmHg})$ & 0.141 & 0.329 \\
\hline RNFLthickness $(\mu \mathrm{m})$ & 0.750 & $\varangle 0.001$ \\
\hline VF MD $(\mathrm{dB})$ & 0.749 & $\varangle 0.001^{\star}$ \\
\hline
\end{tabular}

IOP, intraocular pressure;OPP, ocular perfusion pressure; RNFL, retinal nerve fiber layer; VF MD , visual field mean deviation

Unless otherwise illustrated, correlation analysis was made by using Pearson correlation test.

* Correlation analysis was made by using Spearman correlation test.

\section{Discussion}

There are limited reports on the peripapillary vessel density in PACG. As far as we know, there is no literature reporting the peripapillary vessel density in clock-hour sector in PACG. In this study, we found that the peripapillary vessel density in the mean average, quadrant, and clock-hour sectors of PACG decreased in different degrees compared with normal eyes. The inferior quadrant vessel density had the highest diagnostic value. Except 4 clock-hour, there was not any statistical difference between the diagnostic ability of vessel density and RNFL.

In this study, the peripapillary vessel density of PACG eyes was significantly lower than that of healthy eyes, which were consistent with the studies of Rao et al. [17] and Wang et al.[18]. Rao et al. [17] used OCTA to report the diagnostic ability of peripapillary average vessel density and (divided into 6 parts) each quadrant vessel density parameters in PACG, which were similar to the diagnostic ability of RNFL 
thicknesses. AUCs of average peripapillary vessel density and the inferotemporal vessel density sector were 0.79 and 0.86 respectively. In this study, the average vessel density of PACG was higher than that reported by Rao et al., while the diagnostic ability of RNFL thicknesses was similar. This study showed that the diagnostic ability of vessel density parameters may be better than that of RNFL thicknesses in PACG, although the difference was not statistically significant. The reason may be that the severity of glaucoma in this study (VF mean deviation was - 18.3dB) was much higher than that in Rao et al. (VF mean deviation was -9.2). Previous studies showed that Peripapillary RNFL measured by OCT may not be as effective as visual field examination or macular thickness in monitoring the progression of advanced glaucoma[19, 20]. This result may be partly explained by that RNFL of advanced PACG change slightly, while vessel density continues to decline significantly. In previous studies of PACG, there was no study to evaluate clock-hour vessel density diagnostic ability. In our study, quadrant and clock-hour vascular density had good glaucoma diagnostic ability in all areas.

According to the study on clock-hour sector, we found that the vessel density at 7 o' clock decreased most, and RNFL at 7 o' clock also decreased the most. Previous studies showed that RNFL defects were most often found in infratemporal and supratemporal sectors[21]. Anatomically, RNFL bundles are particularly thick in the superior and inferior quadrants of the optic disc. These areas are particularly vulnerable to glaucoma damage[22]. The sequential relationship between vessel density reduction and RNFL atrophy needs longitudinal study to prove. In our study, the decrease of vessel density was closely related to the decrease of RNFL and the severity of VF mean deviation. This was consistent with the research by Zhang et al[23].

The advantage of this study was that we used imageJ software to calculate the vessel density of each clock sector that matches the clock-hour RNFL sector. We accurately matched clock-hour vessel density sectors and clock-hour RNFL sectors for AUCs diagnostic capability comparison. Our study had some limitations. There was evidence that ocular hypotensive eye drops may affect the hemodynamics of ocular blood flow and retinal vascular autoregulation[24, 25]. PACG eyes were exposed to 0 to 3 antiglaucoma drugs during OCTA examination. Second, the OCTA algorithm we used included large vessels and capillaries, which might not represent the actual loss of capillaries in specific regions. Third, because our study was a cross-sectional study, we cannot determine the cause-effect relationship between vessel density and structure.

Our research showed that OCTA may provide useful information for glaucoma. The diagnostic ability of vessel density in PACG was equivalent to that of traditional RNFL measurement. Combining the information of vessel density measurement may improve the diagnostic rate of glaucoma. Future research can evaluate the application of this new method in detecting glaucoma changes.

In summary, the diagnostic ability of vessel density in PACG is comparable to that of RNFL thickness. Understanding the spatial characteristics of Peripapillary vessel density in PACG may be helpful for clinical diagnosis and treatment. 


\section{Declarations}

\section{Ethics approval and consent to participate}

The study protocol was approved by the Ethics Committee of Joint Shantou International Eye Center (JSIEC) of Shantou University and the Chinese University of Hong Kong (Shantou city, China). The study followed the tenets of the Declaration of Helsinki. Written informed consent was obtained from all subjects. Ethical approval number囚EC20180929(5)-P12

\section{Consent for publication}

Written informed consent has been obtained from the patient.

\section{Availability of data and materials}

The author confirms that all relevant data are included in the article.

\section{Competing interests}

The authors declare that they have no competing interests.

\section{Funding}

This study was supported by the internal funding(10-025) of Joint Shantou International Eye Center of Shantou University and The Chinese University of Hong Kong, Shantou, Guangdong Province, People's Republic of China.

\section{Authors' contributions}

$M Z, Y L, S C$ participated in the design of the study. YL and SC analyzed and interpreted the data. MZ, YL wrote the article. $M Z$ critically revised the article. $Y L, S C$ collected the data. $M Z, Y L$ reviewed the literature. All authors read and approved the final manuscript.

\section{Acknowledgements}

None.

\section{References}

1. Yanagi M, Kawasaki R, Wang JJ, Wong TY, Crowston J, Kiuchi Y: Vascular risk factors in glaucoma: a review. Clinical \& experimental ophthalmology 2011, 39(3):252-258.

2. Liu L, Jia Y, Takusagawa HL, Pechauer AD, Edmunds B, Lombardi L, Davis E, Morrison JC, Huang D: Optical Coherence Tomography Angiography of the Peripapillary Retina in Glaucoma. JAMA ophthalmology 2015, 133(9):1045-1052. 
3. Akil H, Huang AS, Francis BA, Sadda SR, Chopra V: Retinal vessel density from optical coherence tomography angiography to differentiate early glaucoma, pre-perimetric glaucoma and normal eyes. PloS one 2017, 12(2):e0170476.

4. Rabiolo A, Gelormini F, Sacconi R, Cicinelli MV, Triolo G, Bettin P, Nouri-Mahdavi K, Bandello F, Querques G: Comparison of methods to quantify macular and peripapillary vessel density in optical coherence tomography angiography. PloS one 2018, 13(10):e0205773.

5. Richter GM, Chang R, Situ B, Chu Z, Burkemper B, Reznik A, Bedrood S, Kashani AH, Varma R, Wang RK: Diagnostic Performance of Macular Versus Peripapillary Vessel Parameters by Optical Coherence Tomography Angiography for Glaucoma. Translational vision science \& technology 2018, 7(6):21.

6. Yip VCH, Wong HT, Yong VKY, Lim BA, Hee OK, Cheng J, Fu H, Lim C, Tay ELT, Loo-Valdez RG et al: Optical Coherence Tomography Angiography of Optic Disc and Macula Vessel Density in Glaucoma and Healthy Eyes. Journal of glaucoma 2019, 28(1):80-87.

7. Jonas JB, Fernandez MC, Sturmer J: Pattern of glaucomatous neuroretinal rim loss. Ophthalmology 1993, 100(1):63-68.

8. Airaksinen PJ, Tuulonen A, Alanko HI: Rate and pattern of neuroretinal rim area decrease in ocular hypertension and glaucoma. Archives of ophthalmology (Chicago, III: 1960) 1992, 110(2):206-210.

9. Shin JW, Sung KR, Lee JY, Kwon J, Seong M: Optical coherence tomography angiography vessel density mapping at various retinal layers in healthy and normal tension glaucoma eyes. Graefe's archive for clinical and experimental ophthalmology = Albrecht von Graefes Archiv fur klinische und experimentelle Ophthalmologie 2017, 255(6):1193-1202.

10. Tripathi RC, Tripathi BJ, Haggerty C: Drug-induced glaucomas: mechanism and management. Drug Saf 2003, 26(11):749-767.

11. Riva CE, Grunwald JE, Petrig BL: Autoregulation of human retinal blood flow. An investigation with laser Doppler velocimetry. Invest Ophthalmol Vis Sci 1986, 27(12):1706-1712.

12. Foster PJ, Buhrmann R, Quigley HA, Johnson GJ: The definition and classification of glaucoma in prevalence surveys. The British journal of ophthalmology 2002, 86(2):238-242.

13. S C, SC L: Optical Coherence Tomography Angiography in Glaucoma Care. Current eye research 2018, 43(9):1067-1082.

14. Nelis P, Kleffner I, Burg MC, Clemens CR, Alnawaiseh M, Motte J, Marziniak M, Eter N, Alten F: OCTAngiography reveals reduced vessel density in the deep retinal plexus of CADASIL patients. Scientific reports 2018, 8(1):8148.

15. Shoji T, Yoshikawa Y, Kanno J, Ishii H, Ibuki H, Ozaki K, Kimura I, Shinoda K: Reproducibility of Macular Vessel Density Calculations Via Imaging With Two Different Swept-Source Optical Coherence Tomography Angiography Systems. Trans/ Vis Sci Techno/ 2018, 7(6):31.

16. Scripsema NK, Garcia PM, Bavier RD, Chui TY, Krawitz BD, Mo S, Agemy SA, Xu L, Lin YB, Panarelli JF et al: Optical Coherence Tomography Angiography Analysis of Perfused Peripapillary Capillaries in 
Primary Open-Angle Glaucoma and Normal-Tension Glaucoma. Investigative ophthalmology \& visual science 2016, 57(9):Oct611-oct620.

17. Rao HL, Kadambi SV, Weinreb RN, Puttaiah NK, Pradhan ZS, Rao DAS, Kumar RS, Webers CAB, Shetty R: Diagnostic ability of peripapillary vessel density measurements of optical coherence tomography angiography in primary open-angle and angle-closure glaucoma. Br J Ophthalmol 2017, 101(8):1066-1070.

18. Wang $X$, Jiang $C$, Kong $X, Y u X$, Sun $X$ : Peripapillary retinal vessel density in eyes with acute primary angle closure: an optical coherence tomography angiography study. Graefe's archive for clinical and experimental ophthalmology = Albrecht von Graefes Archiv fur klinische und experimentelle Ophthalmologie 2017, 255(5):1013-1018.

19. Leung CK, Liu S, Weinreb RN, Lai G, Ye C, Cheung CY, Pang CP, Tse KK, Lam DS: Evaluation of retinal nerve fiber layer progression in glaucoma a prospective analysis with neuroretinal rim and visual field progression. Ophthalmology 2011, 118(8):1551-1557.

20. Sung KR, Sun JH, Na JH, Lee JY, Lee Y: Progression detection capability of macular thickness in advanced glaucomatous eyes. Ophthalmology 2012, 119(2):308-313.

21. Leung CK, Choi N, Weinreb RN, Liu S, Ye C, Liu L, Lai GW, Lau J, Lam DS: Retinal nerve fiber layer imaging with spectral-domain optical coherence tomography: pattern of RNFL defects in glaucoma. Ophthalmology 2010, 117(12):2337-2344.

22. Hood DC, Raza AS, de Moraes CG, Liebmann JM, Ritch R: Glaucomatous damage of the macula. Prog Retin Eye Res 2013, 32:1-21.

23. Zhang S, Wu C, Liu L, Jia Y, Zhang Y, Zhang Y, Zhang H, Zhong Y, Huang D: Optical Coherence Tomography Angiography of the Peripapillary Retina in Primary Angle-Closure Glaucoma. American journal of ophthalmology 2017, 182:194-200.

24. Tsuda S, Yokoyama Y, Chiba N, Aizawa N, Shiga Y, Yasuda M, Yokokura S, Otomo T, Fuse N, Nakazawa T: Effect of topical tafluprost on optic nerve head blood flow in patients with myopic disc type. J Glaucoma 2013, 22(5):398-403.

25. Feke GT, Bex PJ, Taylor CP, Rhee DJ, Turalba AV, Chen TC, Wand M, Pasquale LR: Effect of brimonidine on retinal vascular autoregulation and short-term visual function in normal tension glaucoma. American journal of ophthalmology 2014, 158(1):105-112.e101.

\section{Figures}



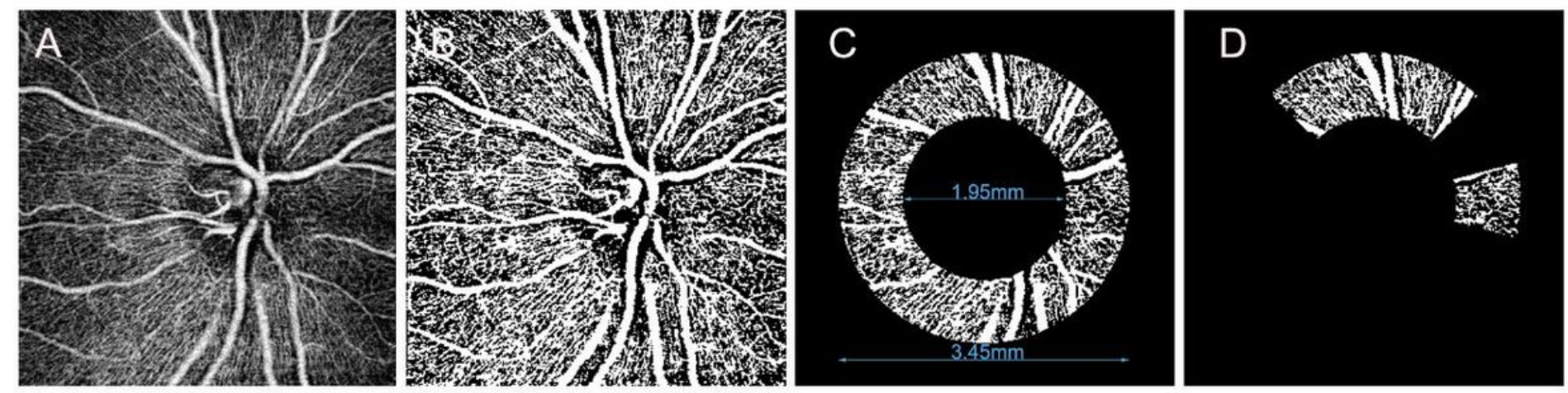

Figure 1

OCT-A image processing steps. (A) Image imported into the Image $\mathrm{J}$ software.(B) Binary image after local adaptive thresholding.(C) Annular region of interest centered at the optic nerve head.(D) Obtaining vessel density values of each quadrant and clock-hour sector
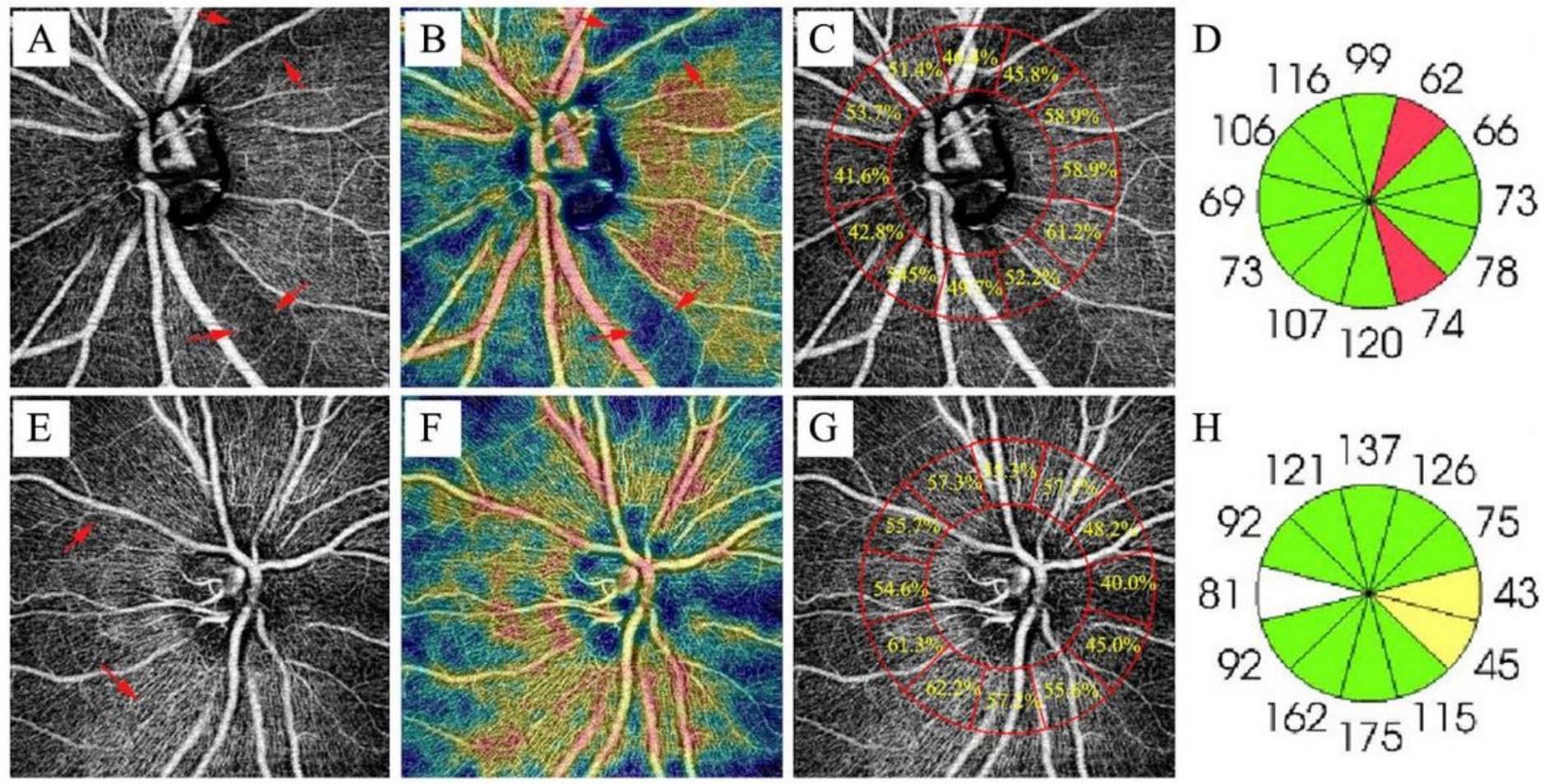

Figure 2

Example of PACG eye with decreased infratemporal and supratemporal vessel density of Optic Disc The infratemporal and supratemporal peripapillary vessel density of glaucoma decreased and the peripapillary retinal nerve fiber layer in corresponding areas atrophied.(first row) On the contrary, these areas were very high in healthy eyes. (second row) (A) Nerve Head scan and (B) Corresponding colorcoded vessel density map of the Nerve Head layer. (C) Vessel density value at clock-hour region (D) Peripapillary retinal nerve fiber layer thickness at clock-hour region. 